\title{
UNIQUENESS AND CONSTRUCTION OF SOLUTION FOR THE NONLINEAR ELASTODIFFUSION PROBLEM
}

BY

\section{XANTHIPPI MARKENSCOFF}

Department of Applied Mechanics and Engineering Sciences, University of California, San Diego, La Jolla, California

I. Introduction. A widely used constitutive equation for nonlinear elastodiffusion [e.g., Hirth (1980), Larche and Cahn (1985), Li, et al. (1996), Lufrano, et al. (1996), and Sofronis (1995)] is

$$
\begin{gathered}
\sigma_{i j}=\lambda \varepsilon_{k k} \delta_{i j}+2 \mu \varepsilon_{i j}-(3 \lambda+2 \mu) \alpha C \delta_{i j}, \\
C=C_{0} e^{\frac{k_{2} \sigma_{t t}}{3 \lambda+2 \mu}},
\end{gathered}
$$

where $C$ denotes the concentration of the diffused atoms, $C_{0}$ the concentration in the absence of stress, $\alpha$ the coefficient of expansion, $k_{2}=\frac{(3 \lambda+2 \mu) \nu_{H}}{3 k T}$ with $\nu_{H}$ being the volume change due to a single solute atom, $k$ is Boltzmann's constant, and $T$ is absolute temperature; $\sigma_{i j}, \varepsilon_{i j}, \lambda, \mu$ are the stress and strain tensors and the Lamé moduli, respectively, and repeated indices imply the summation convention. For an extensive list of references on the above constitutive relation, the reader is referred to the references in the above-mentioned articles.

The elasticity problem under body forces $F_{i} \in L^{2}(\Omega)$, boundary displacements $h_{i} \in$ $H^{1 / 2}(\Omega)$, or tractions $f_{i} \in H^{-1 / 2}(\Omega)$, defined by the equations

$$
\sigma_{i j, j}=F_{i} \quad \text { in } \Omega
$$

with

$$
u_{i}=h_{i} \quad \text { on } \partial \Omega
$$

or

$$
\sigma_{i j} n_{j}=f_{i} \text { on } \partial \Omega
$$

is considered. This is an elasticity problem with the body force depending nonlinearly on the stress. We will prove uniqueness and convergence of the construction of the solution by iteration. Suppose we knew $C \in L^{2}(\Omega)$. Then we can find $u \in H^{1}(\Omega)$ by solving 
(3) with boundary conditions $(4)_{\mathrm{a}}$ or $(4)_{\mathrm{b}}$ regarding $C$ as a body force and/or surface traction. This amounts to solving a boundary value problem of the equations of elasticity.

II. An important identity and uniqueness. Let us assume that two concentrations $C, C^{\prime}$ in $L^{2}(\Omega)$ are given, and let $u_{i}, u_{i}^{\prime}$ be the corresponding displacements in $H^{1}(\Omega)$, with strains $\varepsilon_{i j}, \varepsilon_{i j}^{\prime}$ and stresses $\sigma_{i j}, \sigma_{i j}^{\prime}$, respectively. Then we have

$$
\sigma_{i j, j}=F_{i}, \quad \sigma_{i j, j}^{\prime}=F_{i} \quad \text { in } \Omega,
$$

with

$$
u_{i}=h_{i}, \quad u_{i}^{\prime}=h_{i} \quad \text { on } \partial \Omega
$$

or

$$
\sigma_{i j} n_{j}=f_{i}, \quad \sigma_{i j}^{\prime} n_{j}=f_{i} \quad \text { on } \partial \Omega .
$$

By considering (5), (6), and (1) we write

$$
\begin{aligned}
0= & -\int_{\Omega}\left[u_{i}-u_{i}^{\prime}\right]\left[\sigma_{i j}-\sigma_{i j}^{\prime}\right]_{, j} d x=\int_{\Omega}\left[u_{i}-u_{i, j}^{\prime}\right]_{j}\left[\sigma_{i j}-\sigma_{i j}^{\prime}\right] d x \\
= & \int_{\Omega}\left[\lambda\left(\varepsilon_{k k}-\varepsilon_{k k}^{\prime}\right)^{2}+2 \mu\left(\varepsilon_{i j}-\varepsilon_{i j}^{\prime}\right)\left(\varepsilon_{i j}-\varepsilon_{i j}^{\prime}\right)\right] d x \\
& -\int_{\Omega}(3 \lambda+2 \mu) \alpha\left(C-C^{\prime}\right)\left(\varepsilon_{k k}-\varepsilon_{k k}^{\prime}\right) d x .
\end{aligned}
$$

We note that the inequality

$$
\int_{\Omega}\left\{\lambda\left(\varepsilon_{k k}-\varepsilon_{k k}^{\prime}\right)^{2}+2 \mu\left(\varepsilon_{i j}-\varepsilon_{i j}^{\prime}\right)\left(\varepsilon_{i j}-\varepsilon_{i j}^{\prime}\right)\right\} d x \geq \beta \int_{\Omega}\left(\varepsilon_{k k}-\varepsilon_{k k}^{\prime}\right)^{2} d x
$$

where

$$
\beta= \begin{cases}\frac{3 \lambda+2 \mu}{3} & \text { in all cases, } \\ \lambda+2 \mu & \text { in the case of boundary conditions }(4)_{\mathrm{a}}\end{cases}
$$

holds identically.

From (7), (8), (9), (1), and Schwarz's inequality,

$$
\begin{aligned}
\beta \int_{\Omega}\left(\varepsilon_{k k}-\varepsilon_{k k}^{\prime}\right)^{2} d x & \leq \int_{\Omega}\left[\lambda\left(\varepsilon_{k k}-\varepsilon_{k k}^{\prime}\right)+2 \mu\left(\varepsilon_{i j}-\varepsilon_{i j}^{\prime}\right)\left(\varepsilon_{i j}-\varepsilon_{i j}^{\prime}\right)\right] d x \\
& =(3 \lambda+2 \mu) \alpha \int_{\Omega}\left(C-C^{\prime}\right)\left(\varepsilon_{k k}-\varepsilon_{k k}^{\prime}\right) d x \\
& \leq(3 \lambda+2 \mu) \alpha\left\{\int_{\Omega}\left(C-C^{\prime}\right)^{2} d x\right\}^{1 / 2}\left\{\int_{\Omega}\left(\varepsilon_{k k}-\varepsilon_{k k}^{\prime}\right)^{2} d x\right\}^{1 / 2} .
\end{aligned}
$$

From (10) it follows that

$$
\int_{\Omega}\left(\varepsilon_{k k}-\varepsilon_{k k}^{\prime}\right)^{2} d x \leq \frac{(3 \lambda+2 \mu)^{2} \alpha^{2}}{\beta^{2}} \int_{\Omega}\left(C-C^{\prime}\right)^{2} d x .
$$


From the constitutive equation (2) with $\varepsilon_{k k}=\xi$, it follows that $C \equiv g(\xi)$, so that

$$
\begin{gathered}
\xi=3 \alpha C+\frac{1}{k_{2}} \ln C-\frac{1}{k_{2}} \ln C_{0}, \\
\frac{d \xi}{d C}=3 \alpha+\frac{1}{k_{2} C},
\end{gathered}
$$

and

$$
0 \leq \frac{d g}{d \xi}=\left[3 \alpha+\frac{1}{k_{2} C}\right]^{-1}<\frac{1}{3 \alpha} .
$$

We can observe that (12) is really a consequence of the positive definiteness of the elastic energy. Indeed, setting $C=g\left(\varepsilon_{k k}\right)-g(0)+g(0)$ in (1), we have

$$
\sigma_{i j}=\lambda \varepsilon_{k k} \delta_{i j}+2 \mu \varepsilon_{i j}-(3 \lambda+2 \mu) \alpha\left[g\left(\varepsilon_{k k}\right)-g(0)\right] \delta_{i j}-(3 \lambda+2 \mu) \alpha g(0) \delta_{i j} .
$$

Considering that the last term in (13) is constant hydrostatic pressure, and, by making use of the mean-value theorem, we have for the remaining part that

$$
\begin{aligned}
\int_{\Omega} \sigma_{i j} \varepsilon_{i j} d x & =\int_{\Omega}\left\{\lambda \varepsilon_{k k}^{2}+2 \mu \varepsilon_{i j} \varepsilon_{i j}-(3 \lambda+2 \mu) \alpha\left[g\left(\varepsilon_{k k}\right)-g(0)\right] \varepsilon_{k k} d x\right\} \\
& \geq \int_{\Omega}\left[\frac{3 \lambda+2 \mu}{3} \varepsilon_{k k}^{2}-(3 \lambda+2 \mu) \alpha g^{\prime}(\xi) \varepsilon_{k k}^{2}\right] d x \\
& =\int_{\Omega}(3 \lambda+2 \mu) \varepsilon_{k k}^{2}\left[\frac{1}{3}-\alpha g^{\prime}(\xi)\right] d x
\end{aligned}
$$

which is positive definite if $g^{\prime}<\frac{1}{3 \alpha}$, identical with (12), which makes it clear that the constitutive relation (2) has a strong physical foundation.

Let us assume now that $C$ and $C^{\prime}$ satisfy the constitutive equation (2). Hence, from (12) and the mean-value theorem,

$$
\int_{\Omega}\left(C-C^{\prime}\right)^{2} d x<\frac{1}{9 \alpha^{2}} \int_{\Omega}\left(\varepsilon_{k k}-\varepsilon_{k k}^{\prime}\right)^{2} d x .
$$

Hence, (15) and (11), together with (9), give

$$
\int_{\Omega}\left(\varepsilon_{k k}-\varepsilon_{k k}^{\prime}\right)^{2} d x<\int_{\Omega}\left(\varepsilon_{k k}-\varepsilon_{k k}^{\prime}\right)^{2} d x,
$$

and hence, $\varepsilon_{k k}=\varepsilon_{k k}^{\prime}$, and from (15) also $C=C^{\prime}$. Hence from (10), $\varepsilon_{i j}=\varepsilon_{i j}^{\prime}$, and thus $u_{i}=u_{i}^{\prime}$, and the two solutions coincide, i.e., we proved uniqueness.

\section{Existence and construction of the solution.}

Algorithm. Let us construct sequences $C^{0}, C^{1}, \ldots$ and $u^{1}, u^{2}, \ldots$ as follows:

(a) $C^{0} \equiv 0$.

(b) Given $C^{m} \in L^{2}$, construct $u^{m+1} \in H^{1}$, with strains $\varepsilon_{i j}^{m+1}$, by solving (5) and (6) with $C$ assumed known, equal to $C^{m}$.

(c) Compute $C^{m+1}$ through

$$
C^{m+1}=g\left(\varepsilon_{k k}^{m+1}\right) .
$$

Note that $C^{m+1} \in L^{2}(\Omega)$ because of (12). 
We claim that

$$
\begin{gathered}
\left(u^{m}, C^{m}\right) \rightarrow(u, C), \\
u^{m} \stackrel{H^{1}}{\longrightarrow} u, \quad C^{m} \stackrel{L^{2}}{\longrightarrow} C
\end{gathered}
$$

and thus $(u, C)$ is a solution of our boundary value problem (fixed point).

To prove convergence of the above algorithm, in the case of the boundary value problem $(6)_{\mathrm{a}}$, no further assumption is necessary. In the case of the boundary value problem $(6)_{\mathrm{b}}$, the assumption is that $C$ is pointwise bounded, $C \leq M$, or, equivalently, $\varepsilon_{k k}$ is pointwise bounded. Indeed,

$$
\int_{\Omega}\left(\varepsilon_{k k}^{m+1}-\varepsilon_{k k}^{m}\right)^{2} \leq \frac{(3 \lambda+2 \mu)^{2}}{\beta^{2}} \alpha^{2} \int_{\Omega}\left(C^{m}-C^{m-1}\right)^{2} d x .
$$

From this we need to derive

$$
\int_{\Omega}\left(\varepsilon_{k k}^{m+1}-\varepsilon_{k k}^{m}\right)^{2} d x \leq \gamma^{2} \int\left(\varepsilon_{k k}^{m}-\varepsilon_{k k}^{m-1}\right)^{2} d x
$$

where $\gamma<1$.

Now, in the case of the boundary value problem (6) a, we use $\beta=\lambda+2 \mu$, from (9). Using also (12) and the mean-value theorem, from (17) it follows that

$$
\int_{\Omega}\left(\varepsilon_{k k}^{m+1}-\varepsilon_{k k}^{m}\right)^{2} d x \leq \frac{(3 \lambda+2 \mu)}{(\lambda+2 \mu)^{2}} \alpha^{2} \frac{1}{9 \alpha^{2}} \int_{\Omega}\left(\varepsilon_{k k}^{m}-\varepsilon_{k k}^{m-1}\right)^{2} d x .
$$

Note that $\gamma=\frac{3 \lambda+2 \mu}{3(\lambda+2 \mu)}<1$.

In the case of the boundary value problem $(6)_{\mathrm{b}}, \beta=\frac{3 \lambda+2 \mu}{3}$. However, because of the assumption $C \leq M$, from (12),

$$
\frac{d g}{d \xi} \leq\left(3 \alpha+\frac{1}{k_{2} M}\right)^{-1}
$$

Then (17) with the use of the mean-value theorem yields

$$
\int_{\Omega}\left(\varepsilon_{k k}^{m+1}-\varepsilon_{k k}^{m}\right)^{2} d x \leq \frac{(3 \lambda+2 \mu)^{2} \alpha^{2}}{\frac{(3 \lambda+2 \mu)^{2}}{9}}\left[3 \alpha+\frac{1}{k_{2} M}\right]^{-1} \int_{\Omega}\left(\varepsilon_{k k}^{m}-\varepsilon_{k k}^{m-1}\right)^{2} d x .
$$

Hence $\gamma=\frac{1}{1+\frac{1}{3 k_{2} M \alpha}}<1$.

Thus (18) has been established, and it follows that $\varepsilon_{k k}^{m} \rightarrow \varepsilon_{k k}$ in $L^{2}$. Then the meanvalue theorem implies $C^{m} \rightarrow C$ in $L^{2}$. Then $\varepsilon_{i j}^{m} \rightarrow \varepsilon_{i j}$ in $L^{2}$ and hence $u^{m} \rightarrow u$ in $H^{1}$.

We have thus proved that the solution exists and can be constructed by iteration; it is also unique. The good behavior of this problem is due to the fact that the concentration law (2) is physically founded and consistent with positive definiteness (pointwise) requirements of the energy. The limit is actually sharper for the boundary value problem of displacement if we look for positiveness in the integral sense.

The above procedure also addresses the question of whether the solution may converge near singular points of the stress, such as near crack tips. The answer is positive for the boundary value problem of displacement. For the boundary value problem of traction, if 
$\varepsilon_{k k}$ is not pointwise bounded, then the iteration will not converge, and this may be the case at crack-tips.

IV. Extension to a more general body force function. The previous problem may be generalized to a more general constitutive relationship where, instead of (1), we have

$$
\sigma_{i j}=\lambda \varepsilon_{k k} \delta_{i j}+2 \mu \varepsilon_{i j}-F_{i j}\left(\varepsilon_{i j}\right) .
$$

The analysis follows a similar procedure, and (7) now becomes

$$
\begin{aligned}
0= & -\int_{\Omega}\left[u_{i}-u_{i}^{\prime}\right]\left[\sigma_{i j}-\sigma_{i j}^{\prime}\right]_{, j} d x=\int_{\Omega}\left[u_{i}-u_{i}^{\prime}\right]_{, j}\left[\sigma_{i j}-\sigma_{i j}^{\prime}\right] d x \\
= & \int_{\Omega}\left\{\lambda\left(\varepsilon_{k k}-\varepsilon_{k k}^{\prime}\right)^{2}+2 \mu\left(\varepsilon_{i j}-\varepsilon_{i j}^{\prime}\right)\left(\varepsilon_{i j}-\varepsilon_{i j}^{\prime}\right)\right\} d x \\
& -\int_{\Omega}\left(\varepsilon_{i j}-\varepsilon_{i j}^{\prime}\right)\left[F_{i j}\left(\varepsilon_{i j}\right)-F_{i j}\left(\varepsilon_{i j}^{\prime}\right)\right] d x ;
\end{aligned}
$$

from (22) and the inequality (8), as before, it follows that

$$
\int_{\Omega}\left[\varepsilon_{i j}-\varepsilon_{i j}^{\prime}\right]\left[F_{i j}\left(\varepsilon_{r s}\right)-F_{i j}\left(\varepsilon_{r s}^{\prime}\right)\right] d x \geq \beta \int_{\Omega}\left(\varepsilon_{i j}-\varepsilon_{i j}^{\prime}\right)^{2} d x .
$$

Proceeding as before we use the mean-value theorem

$$
\left|F_{i j}\left(\varepsilon_{r s}\right)-F_{i j}\left(\varepsilon_{r s}^{\prime}\right)\right| \leq\|\nabla F\|\left|\varepsilon_{i j}-\varepsilon^{\prime}\right|
$$

where $\|\nabla F\|$ is the maximum norm of the gradient in the domain.

From (23) and (24) it follows that

$$
\|\nabla F\| \int_{\Omega}\left(\varepsilon_{i j}-\varepsilon_{i j}^{\prime}\right)^{2} d x \geq \beta \int_{\Omega}\left(\varepsilon_{i j}-\varepsilon_{i j}^{\prime}\right)^{2} d x,
$$

so that for contraction we require

$$
\frac{\beta}{\|\nabla F\|}<1
$$

We may note that, in addition to uniqueness and existence, stability of the solution with respect to perturbation of the body force $F_{i}$ or the boundary conditions $h_{i}, f_{i}$, can also be established following the above procedure.

Acknowledgments. This research was funded by the National Science Foundation.

\section{REFERENCES}

[1] J. P. Hirth, Effects of hydrogen on the properties of iron and steel, Met. Trans. 11A, 861-890 (1980)

[2] F. C. Larche and J. W. Cahn, The interaction of composition and stress in crystalline solids, Acta Metall. 33, 331-357 (1985)

[3] J. C. M. Li, R. Oriani, and L. S. Darken, The thermodynamics of stressed solids, Z. Physik Chem. Neue Folge 49, 271-291 (1996)

[4] J. Lufrano, P. Sofronis, and H. K. Birnbaum, Modeling of hydrogen transport and elastically accommodated hydride formation near a crack tip, J. Mech. Phys. Sol. 44, 179-205 (1996)

[5] P. Sofronis, The influence of mobility of dissolved hydrogen on the elastic response of a metal, J. Mech. Phys. Sol. 43, 1385-1407 (1995) 